\title{
The magnetic structure of a thin ferromagnetic film on the rough surface of an antiferromagnet
}

Cite as: Low Temp. Phys. 37, 866 (2011); https://doi.org/10.1063/1.3671860

Published Online: 30 December 2011

A. S. Kovalev, and M. L. Pankratova

\section{ARTICLES YOU MAY BE INTERESTED IN}

Properties of ferromagnetic film hysteresis, on the surface of a hard-magnetic antiferromagnet, with a domain structure

Low Temperature Physics 40, 990 (2014); https://doi.org/10.1063/1.4901921

LOW TEMPERATURE TECHNIQUES OPTICAL CAVI『V PHVSICS MITIGATING THERMAL \& VIBRATIONAL NOISE

\section{DOWNLOAD THE WHITE PAPER}




\title{
The magnetic structure of a thin ferromagnetic film on the rough surface of an antiferromagnet
}

\author{
A. S. Kovaleva) and M. L. Pankratova \\ B. Verkin Institute for Low Temperature Physics and Engineering of the National Academy of Sciences of \\ Ukraine, 47 Lenin Ave., Kharkov 61103, Ukraine
}

(Submitted April 21, 2011)

Fiz. Nizk. Temp. 37, 1085-1091 (September-October 2011)

The magnetic structure (including domanization) of a thin ferromagnetic film in contact with a rigid antiferromagnet is investigated under the assumption that the interface is rough and contains individual atomic steps of different signs, isolated "dipole of the steps," and a space-ordered periodic system of steps of alternate signs. (C) 2011 American Institute of Physics. [doi: $10.1063 / 1.3671860$ ]

The problem of coexistence of ferromagnetic (FM) and antiferromagnetic (AFM) phases first occurred in the study of $\mathrm{Co} / \mathrm{CoO}$ alloys ${ }^{1}$ and was first investigated theoretically in $1962 .^{2}$ Currently due to the practical use of the giant magnetoresistance phenomenon in the information reading elements magnetic multilayer FM/AFM nanosystems are widely investigated. As a rule, in the theoretical description of the interface simple classical models of magnets in the exchange approximation are used, and layers of a thickness of several interatomic distances are considered. ${ }^{3,4}$ Special attention is drawn to the "exchange bias" (EB) of these systems. 5,6 Some experimentally observed features of this phenomenon, that have been observed experimentally, ${ }^{7}$ have been investigated theoretically in the previous works. ${ }^{8,9}$ These features were considered to be related to the emergence of inhomogeneous states in the FM subsystem of unfilled domain boundaries (DB), parallel to the interfaces. At the same time, it was suggested that AFM is "magnetically rigid" and layered, and the ordered layers are parallel to the interface. Thus, it was believed that the interface is uncompensated, and the local field, applied from the AFM side on the FM subsystem, leads to EB. However, in some experiments EB was observed in a compensated interface (for example, in the case of AFM ordered in a "checkered" pattern). Numerical experiments would point to the fact that in this case inhomogeneous states of the DB type appear and are oriented perpendicular to the interface. In this work it is shown that this phenomenon is possible in the case of a layered AFM as a result of roughness of the interface. This roughness can be modeled by a sequence of atomic steps of alternating signs on the interface. As shown previously, ${ }^{10-12}$ every such step is related to a "halfvortices" type disturbance that turns into a DB, perpendicular to the interface, in the magnetic structure of the FM.

\section{THE MAGNETIC STRUCTURE OF FERROMAGNETIC FILMS IN CONTACT WITH THE AFM IN THE PRESENCE OF ATOMIC STEPS AT THE INTERFACE}

Let us consider the continuous model of a FM film of thickness $h \gg a$ (where $a$ is interatomic distance) in contact with a rigid layered AFM (consider the orientation of AFM magnetic moments to be fixed). Presence of large easy-plane anisotropy, "shaping" the magnetic moments into a plane, is assumed. In this plane their orientation is defined only by their angle of rotation $\varphi(x, z)$ (scalar model) in the easy plane, where $x$ is the coordinate running along the interface, and $z$ is the perpendicular one. In the case of isotropy of magnetic properties in the easy plane redistribution of magnetization has the form of a magnetic vortex (Fig. 1(a)), while if magnetic anisotropy in the easy plane is considered (biaxial magnetic) this distribution is transformed into a DB, perpendicular to the interface (Fig. 1(b)).

In the isotropic case in the long wavelength limit, when the exchange energy can be expressed as $E_{\mathrm{ex}}$ $=\int d x d z(J / 2)(\nabla \varphi)^{2}$, magnetization distribution is defined by the Laplace equation:

$$
\frac{\partial^{2} \varphi}{\partial x^{2}}+\frac{\partial^{2} \varphi}{\partial z^{2}}=0, \quad z<0
$$

supplemented by boundary conditions on the surface of a ferromagnetic $\partial \varphi /\left.\partial z\right|_{z=-h}=0$ and a condition at the interface. Since we assume that exchange interaction through the interface, which is characterized by exchange constant $J_{0}$, has the same order as exchange interaction in the bulk FM with a constant $J$, then just the quadratic terms can be left in the interaction energy through the interface, and the boundary condition can be written as

$$
\left.\frac{\partial \varphi}{\partial z}\right|_{z=0}=-\frac{J_{0}}{J a}\left(\varphi_{0}(x)-\tilde{\varphi}(x)\right),
$$

where $\varphi_{0}(x)$ is the distribution of magnetization on the interface in a FM, and $\tilde{\varphi}(x)$ is the fixed distribution of magnetization on the interface in an AFM. As $\tilde{\varphi}(x)$ it is convenient to select function $\tilde{\varphi}(x)=2 \operatorname{arctg} \exp (\mu x)$ with $\mu \sim 1 / a$ (let $\mu=2 / \pi a)$. ((In the limit $\mu \rightarrow \infty$ we have $\tilde{\varphi}(x)=\pi \theta(x)$, where $\theta(x)$ is a step function.) We now turn to a new field variable $u(x, z)=\partial \varphi / \partial x$, for which Eq. (1) and the boundary condition at the free boundary retain their form, and in the boundary condition (2) for $\partial u / \partial z$ and $u_{0}$ instead of $\tilde{\varphi}(x)$ there is the function $g(x)=\mu / \operatorname{ch}(\mu x)$, that transforms into $\delta(x)$ in the limit $\mu \rightarrow \infty$.

In terms of the variable $u(x, z)$ solution of Eq. (1), satisfying the boundary conditions at the free boundary of the FM, has the form 
$a$

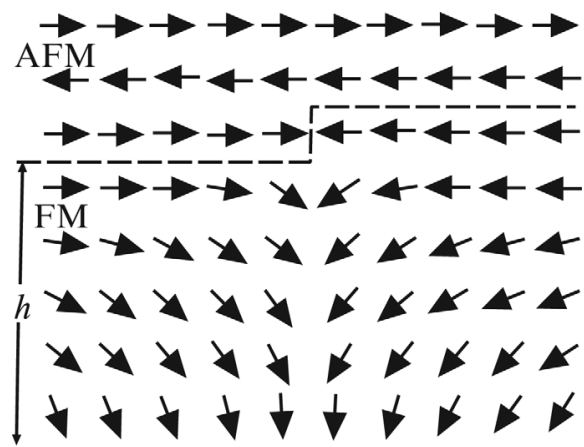

$b$

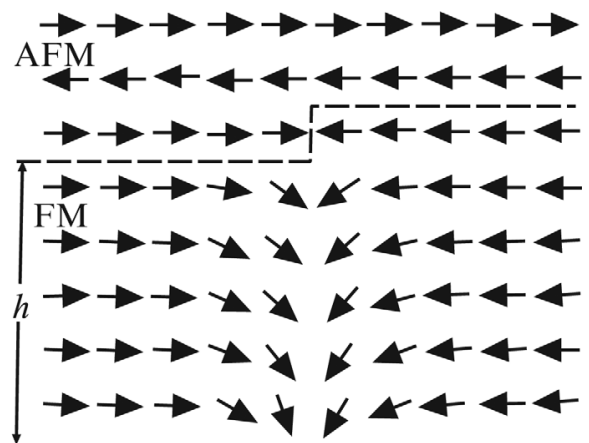

FIG. 1. Magnetization distribution in the FM film that is in contact with a rigid layered AFM in the presence of an atomic step at the interface: in absence of magnetic anisotropy (a), and when anisotropy is taken into consideration (b).

$$
u(x, z)=\int_{0}^{\infty} d \lambda \operatorname{ch} \lambda(z+h) \cos (\lambda x) u(\lambda) .
$$

Substituting Eq. (3) into the boundary condition on the interface we obtain the final expression for the distribution of magnetization in the FM:

$$
u(x, z)=\int_{0}^{\infty} \frac{\cos (\lambda x) \operatorname{ch}(\lambda(z+h)) d \lambda}{\operatorname{ch}(\varepsilon \lambda h) \operatorname{ch}(\lambda h)\left[1+\varepsilon\left(J a / J_{0}\right) \lambda h \operatorname{th}(\lambda h)\right]},
$$

where $\varepsilon=a / h \ll 1$. In the bulk of the FM (at $z \gg a$ ) x-kernel of the Fourier transform approximately reduces to the expression $\operatorname{ch}(\lambda(h-|z|)) / \operatorname{ch}(\lambda h)$ and in this region the solution takes the form

$u(x, z) \approx \frac{\pi}{h} \cos \frac{\pi}{2}\left(1-\frac{|z|}{h}\right) \operatorname{ch} \frac{\pi}{2} \frac{x}{h}\left[\cos \pi\left(1-\frac{|z|}{h}\right)+\operatorname{ch} \pi \frac{\pi}{h}\right]^{-1}$.

At the free boundary $u(x, z=-h)=\pi /(2 h \operatorname{ch}(\pi x / 2 h))$ and the magnetization distribution is transformed to

$$
\varphi(x, z=-h) \approx 2 \operatorname{arctg} \exp (\pi x / 2 h),
$$

and at $z \rightarrow 0$ from Eq. (3) we have $\varphi \rightarrow \tilde{\varphi}=2 \operatorname{arctg} \exp (\pi x / 2 a)$. Thus, as distance from the interface increases from zero to $h$ region of localization of magnetization distribution inhomogeneity increases from $a$ to $h$.

Let us consider the effect of additional magnetic anisotropy in the easy plane. Its energy is given by $E_{\text {an }}=-(\beta / 2)$ $\cos ^{2} \varphi$. (It is assumed that the ground state direction of the easy axis in the plane coincides with the $x$-axis.) Single-ion anisotropy constant $\beta$, as a rule, is much smaller than the exchange constant $J$. Note that in this thin film ferromagnet an additional easy-plane anisotropy with an effective constant $\beta_{\mathrm{ef}}=4 \pi$ emerges due to the magnetic dipole interaction. In view of the magnetic anisotropy equation (1) transforms to the static two-dimentional sine Gordon equation:

$$
\frac{\partial^{2} \varphi}{\partial x^{2}}+\frac{\partial^{2} \varphi}{\partial z^{2}}-\frac{1}{2 l^{2}} \sin 2 \varphi=0, \quad z<0
$$

where $\sqrt{J / \beta}-l$ is the magnetic length of the ferromagnet. In the infinite medium solution of this equation for the DB, parallel to the $z$-axis, has the form

$$
\varphi=2 \operatorname{arctg} \exp \left(\frac{x}{l}\right) .
$$

In order to qualitatively analyze the transformation of the magnetization distribution near the step at the interface ("half" of the magnetic vortex) in the DB in the bulk of the ferromagnet, consider the model in which a piecewise parabolic function is chosen as the anisotropy energy (piece-wise linear model of the Klein-Gordon equation): $E_{\text {an }}=(\beta / 2) \varphi^{2}$ when $0<\varphi<\pi / 2$ and $E_{\text {an }}=(\beta / 2)(\pi-\varphi)^{2}$ when $\pi / 2<\varphi$ $<\pi$. Equation (7) is transformed into a static Klein-Gordon equation:

$$
\frac{\partial^{2} \varphi}{\partial x^{2}}+\frac{\partial^{2} \varphi}{\partial z^{2}}-\frac{\varphi}{l^{2}}=0, \quad z<0, \quad x<0,
$$

while Eqs. (3) and (4) are transformed in the following manner:

$$
\begin{aligned}
u(x, z) & =\int_{0}^{\infty} d \lambda \operatorname{ch} \tilde{\lambda}(z+h) \cos (\lambda x) u(\lambda) \\
& =\int_{0}^{\infty} \frac{\cos (\lambda x) \operatorname{ch}(\tilde{\lambda}(z+h)) d \lambda}{\operatorname{ch}(\varepsilon \lambda h) \operatorname{ch}(\tilde{\lambda} h)\left[1+\varepsilon\left(J a / J_{0}\right) \tilde{\lambda} h \operatorname{th}(\tilde{\lambda} h)\right]},
\end{aligned}
$$

where $\tilde{\lambda}=\sqrt{\lambda^{2}+1 / l^{2}}$. At the free boundary of the FM film when $h \gg a$ solution has the form $u(x, z=-h)$ $\approx \int_{0}^{\infty} d \lambda \cos \lambda x \sec h(\tilde{\lambda} h)$. The asymptotics of this expression at large distances is determined by the Fourier transform for small values of $\lambda$. Expanding $\sec h(\tilde{\lambda} h)$ into a series for small values of $\lambda$ up to terms $\sim \lambda^{2}$, we obtain the asymptotics of solutions as $x \rightarrow \infty$ (for comparison, asymptotics for an isotropic FM and for DB in an infinite anisotropic ferromagnet with $|z|=h$ are given below):

$$
u \approx \frac{2}{l} \exp \left(-\frac{x}{l}\right), \text { infinite anisotropic ferromagnet, }
$$

$u \approx \frac{\pi}{h} \exp \left(-\frac{\pi}{2} \frac{x}{h}\right)$, film of an isotropic ferromagnet,

$$
u \approx \frac{\pi l}{\sqrt{2 h} \sqrt{l^{2}+h^{2}}} \exp \left(-\sqrt{2} \sqrt{1+\frac{h^{2}}{l^{2}}} \frac{x}{h}\right),
$$

film of an anisitropic ferromagnet.

Comparison of these expressions shows that the domain wall forms at distance $l$ from the step at the interface, while when 
$|z|<l$ distribution of magnetization displays vortex character. Qualitatively, magnetization distribution in anisotropic ferromagnetic film is shown in Figure 1(b).

\section{THE MAGNETIC STRUCTURE OF A FERROMAGNETIC FILM IN CONTACT WITH THE AFM IN THE PRESENCE OF A ROUGH INTERFACE}

Consider the simplest model of a rough interface surface between FM/AFM, in which the heterogeneity of the interface appears as a periodic sequence of atomic steps of alternating sign. We assume that the roughness is weak (low density of steps) and the distance between the steps $L$ is significantly greater than the magnetic length: $L \gg l$. In addition, we assume that the thickness of the FM film $h \gg l$, and in the film of the anisotropic FM domain walls form, which are related to the steps at the interface. If the steps are of the same sign $s=\left(z_{0}(x>0)-z_{0}(x<0)\right) / a= \pm 1$, where $z_{0}$ is the interface coordinate, there are two different magnetization distributions of the vortex type in its vicinity (see Fig. 2) with different values of the topological charge $\sigma=(1 / 2 \pi)$ $\oint d \chi \varphi(\chi)= \pm 1$, where $\chi$ is the azimuthal angle of the step bypass. Since for a given sign $s$ arbitrary values $\sigma= \pm 1$ are possible, an arbitrary alternation of the vortices and antivortices at the interface is also possible. Fig. 3 shows the simplest configurations with a sequence of vortices (Fig. 3(a)) and the regular alternation of vortices and antivortices (Fig. 3(b)). When vortices are in sequence a regular periodic sequence of domain boundaries of the same sign, which trepel each other, is formed in the bulk of the FM. Configuration of domain walls (DW), perpendicular to the interface, is stable. Prom a physicist's point of view, more interesting is the case when vortices and antivortices associated with the steps at the interface alternate periodically. In a strong magnetic field applied along the anisotropy axis and a smaller magnetic field of spin-slope in the AFM, magnetization of the FM is directed along it everywhere in the bulk. When the magnitude of the field near the surface is decreased closed

$a$
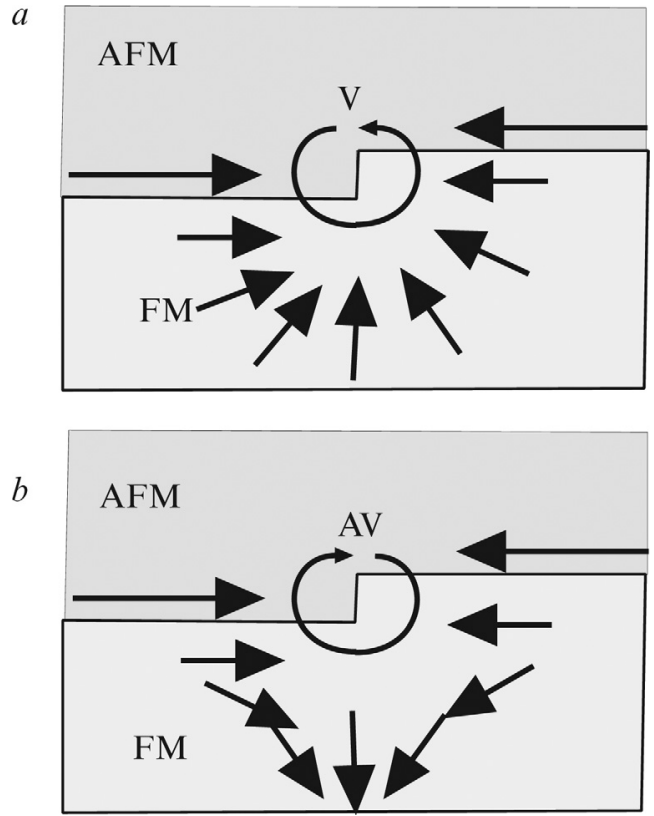

FIG. 2. Magnetization distribution in FM subsystem near atomic step at the interface: vortex configuration (V), $\sigma=1$ (a), and antivortex configuration $(\mathrm{AV}), \sigma=-1$ (b). $a$

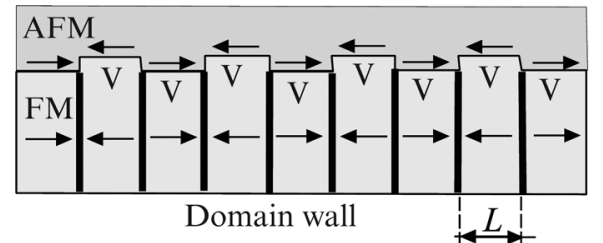

$\varphi$, radians
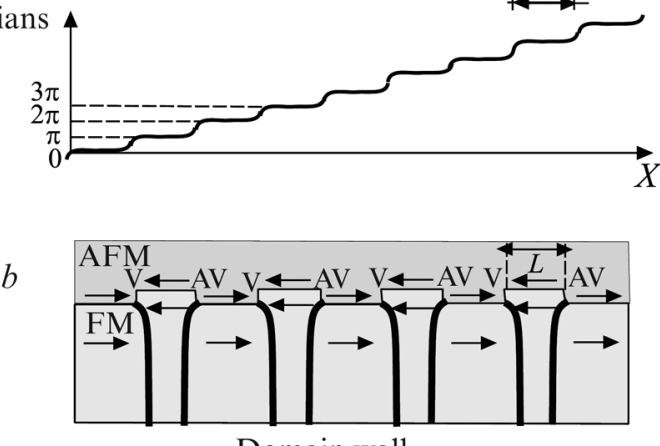

$\varphi$, radians

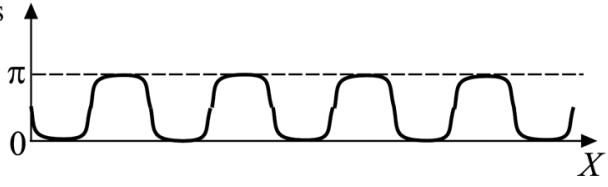

FIG. 3. Domain structure of the FM film near a step interface: (a) when the vortex system at the interface is periodic and (b) when vortices and antivortices alternate regularly at the interface.

domains form that are bounded by the DB, connecting the two nearest atomic steps of different signs (see Fig. 4(a)). Figure shows that this results in formation of the vortex antivortex pair. With further decrease in the field magnitude closed domains "germinate" through the bulk of the FM film, forming in the zero field a structure, in which the number of vortices coincides with the number of antivortices, in particular, of the type shown in Fig. 3(b).

Consider the configuration of the domain structure in a regular alternation of vortices and antivortices at the FM/ AFM interface. Due to the interaction of the closest DB they take on a curved shape that is determined by competition of

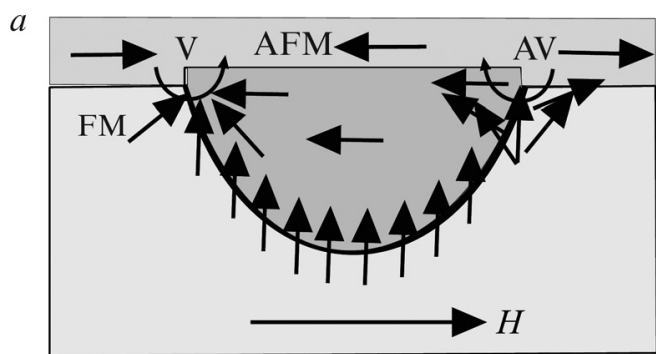

$b$

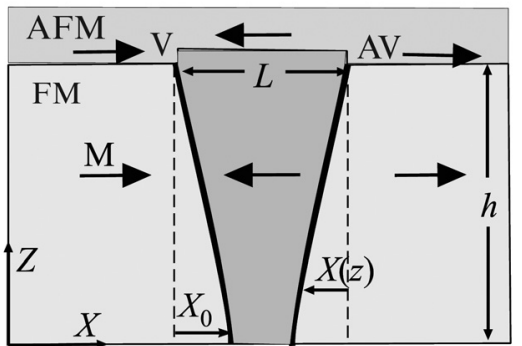

FIG. 4. Associated with the surface dipole of an isolated step in the domain of the FM film: (a) domain in an external magnetic field with a trailing domain boundary (DB), connecting the vortex and antivortex at the interface, and (b) domain in the absence of external field in the case when $h \gg L$. 
attraction energies between DBs and the change in energy of DB itself due to an increase in its length. As you know, parallel domain walls of different signs attract and their attractive energy (per unit of length of $\mathrm{DB}$ ) is equal to $E_{\text {int }}=-E_{0} / \mathrm{ch}^{2}(\Delta X / 2 l)$, where $\Delta X$ is the distance between $\mathrm{DBs},{ }^{13}$ and decreases exponentially at large distances: $E_{\text {int }} \approx-4 E_{0} \exp (-\Delta X / l)$, where $E_{0}=2 \sqrt{J \beta}$ is the unit of length of DB. Since the attractive energy quickly decreases with increasing distance, it is natural to assume that for curved DBs in the given equation the following substitution can be made: $\Delta X \rightarrow \Delta X(z)$. Let us check this assumption.

We compute the interaction of weakly curved DBs. We use the piecewise parabolic model for the magnetic anisotropic energy. In this model an isolated domain wall, oriented along the $z$-axis and having slight deviations $X(z)$ from the main straight-line state of its center along the $x$-axis, is described by Eq. (9), defined for the entire axis $-\infty<x<\infty$ and containing on the right side the following expression: $\pi \theta$ $(X(z)-x) / l^{2}$. Function $u=d \varphi / d x$ introduced earlier also satisfies the static Klein-Gordon equation with the $\delta$-function on the right side:

$$
\frac{\partial^{2} u}{\partial x^{2}}+\frac{\partial^{2} u}{\partial z^{2}}-\frac{u}{l^{2}}=\frac{\pi}{l^{2}} \delta(X(z)-x) .
$$

The Green's function of this equation in an infinite system is well known:

$$
G\left(x-x_{0}, z-z_{0}\right)=2 K_{0}\left(\sqrt{\left(x-x_{0}\right)^{2}+\left(z-z_{0}\right)^{2}} / l\right)
$$

where $K_{0}(p)$ is the modified Bessel function. Because it decreases exponentially rapidly with distance, then, neglecting the edge effects at distances $\delta z \sim l$ from the boundaries, magnetization deformation caused by the curved DB at large distances can be approximately represented as

$$
\begin{aligned}
u(x, z) \approx & \frac{\sqrt{\pi}}{2 l \sqrt{2 l}} \int_{0}^{h} d z^{\prime}\left(\left(x-X\left(z^{\prime}\right)\right)^{2}+\left(z-z^{\prime}\right)^{2}\right)^{-1 / 4} \\
& \times \exp \left(-\frac{1}{l} \sqrt{\left(x-X\left(z^{\prime}\right)\right)^{2}+\left(z-z^{\prime}\right)^{2}}\right) .
\end{aligned}
$$

Let us calculate the interaction energy between two slightlycurved DBs of different signs, found at average distance $L$ from each other. If $\varphi_{1,2}$ are magnetization fields of two walls, then energy of their interaction is equal to

$$
E_{\text {int }}=2 J \int_{0}^{h} d z_{2} \int d x\left(\left(d \varphi_{1} / d x\right)\left(d \varphi_{2} / d x\right)+\varphi_{1} \varphi_{2} / l^{2}\right),
$$

where integration is carried out near the second wall. For magnetization of the second DB we can approximately take the function: $\varphi_{2}=0$ when $x>L+X_{2}\left(z_{2}\right)+l / 2, \varphi_{2}=\pi$ when $x<L+X_{2}\left(z_{2}\right)-l / 2$, and $\varphi_{2}=-\left(x-L-X_{2}\left(z_{2}\right)\right) \pi / l+\pi / 2$ when $-l / 2<x-L-X_{2}<l / 2$. Then, subject to the natural inequalities $L \gg l$ and $L \gg X_{2}\left(z_{2}\right)-X_{1}\left(z_{1}\right) \gg l$, where $X_{n}\left(z_{n}\right)$ are coordinates of DB centers at point $z_{n}$, at an arbitrary ratio of parameters $L$ and $h$ interaction energy of two DBs is reduced to the expression

$$
\begin{aligned}
E_{\text {int }} \approx & -E_{0} \frac{\pi \sqrt{\pi}}{4 \sqrt{2}} \frac{1}{\sqrt{l L}} \int_{0}^{h} d z_{1} \int_{0}^{h} d z_{2} \\
& \times \exp \left\{-\frac{1}{l} \sqrt{\left(L+X_{2}\left(z_{2}\right)-X_{1}\left(z_{1}\right)\right)^{2}+\left(z_{2}-z_{1}\right)^{2}}\right\} .
\end{aligned}
$$

The exponent in this formula decreases by $e$ times at $z_{2}-z_{1} \sim 2 L l$, and the terms in the radicans are of the order of magnitude of $L^{2}, L\left(X_{2}-X_{1}\right)$ and $L l$. Therefore, when inequalities above are true, energy Eq. (16) takes the form

$$
E_{\mathrm{int}} \approx-(\pi / 2)^{2} E_{0} \int d z \exp [-(L-2 X(z)) / l]
$$

Since $L-2 X(z)=\Delta X(z)$ (and $\Delta X(z)$ is the distance between $\mathrm{DBs})$ in the case of parallel linear dislocations, this expression agrees qualitatively with the above. (It is considered that because of symmetry considerations $X_{2}(z)$ $=-X_{1}(z)$.) The difference by a factor of 1.5 between the numerical coefficients is due to the use of different models: the sine Gordon model and the piecewise linear model of the Klein-Gordon equation. In the case of correct alternation of the vortex and antivortex features at the interface distances between the DBs at a fixed value of $z$ equal to $L \pm 2 X(z)$, and the estimated energy per DB is:

$$
E_{\mathrm{int}} \approx-2 E_{0} \int_{0}^{h} d z \exp \left(-\frac{L}{l}\right) \operatorname{ch} \frac{2 X(z)}{l} .
$$

Length of the curved DB is

$$
\lambda=\int_{0}^{h} d z \sqrt{1+(d X(z) / d z)^{2}}
$$

and excess energy is determined by the formula

$$
E_{\mathrm{cur}} \approx \frac{E_{0}}{2} \int_{0}^{h} d z\left(\frac{d X(z)}{d z}\right)^{2}
$$

Thus, the total additional energy of one DB of the periodic domain structure associated with the bending of the DB equals to

$$
\Delta E \approx E_{0} \int_{0}^{h} d z\left[\frac{1}{2}\left(\frac{d X}{d z}\right)^{2}-2 \exp \left(-\frac{L}{l}\right) \operatorname{ch}\left(2 \frac{X}{l}\right)\right],
$$

and the corresponding equation for the shape of the curved DB has the form

$$
\frac{d^{2} X}{d z^{2}}+\frac{4}{l} \mathrm{e}^{-L / l} \operatorname{sh}\left(2 \frac{X}{l}\right)=0
$$

with the boundary conditions at the boundaries of the FM film: $\left.X\right|_{z=0}=0, d X /\left.d z\right|_{z=-h}=0$. Equation (20) has the following solution that satisfies the specified boundary conditions:

$$
X(z)=l \operatorname{Arcch}\left(1 / \operatorname{dn}\left(\frac{z}{h} K(k), k\right)\right),
$$

where $d n(p, k)$ is the Jacobi elliptic function, $K(k)$ is the complete elliptic integral of the first kind, and the modulus of the elliptic function $k$ is defined by the equation 


$$
\sqrt{1-k^{2}} K(k)=2 \sqrt{2} \frac{h}{l} \exp \left(-\frac{L}{2 l}\right)
$$

and is associated with maximal displacement of the DB at the free boundary of ferromagnetic films: $k=\operatorname{th}(X(h) / l)$. From the latter we see that for $X(h) \gg l$ the modulus of the elliptic function $k \approx 1$ and $k^{\prime}=\sqrt{1-k^{2}} \ll 1$. The entire curve of the DB is concentrated in the area $\Delta z \sim h l /$ $X(h)=l \exp (L / 2 l) \ll h$, which is significantly greater that the thickness of the layer near the interface $\delta z \sim l$, where transformation of the magnetization vortex distribution into a formed DB occurs. In the most fascinating from a physicist's point of view case, when $l \ll X(h) \ll L$ and the effect of DB bending manifests itself, but the shift is small compared to the distance between the walls, there is the following restriction on the thickness of the FM film: $l \exp (L / 2 l) \ll h \ll L$ $\exp (L / 2 l)$. When these inequalities are true, the solution of Eq. (21) in a narrow region near the boundary $z<l$ is approximately reduced to a linear dependence $X(z) \approx$ $z X(h) / h$, then near $\Delta z$ a fast access to the limiting value $X(h) \approx h \exp (-L / 2 l)$ is observed. (The deviation from this value in the bulk of the FM plate is insignificant: $X(z) \approx X(h)-\left(X(h)^{2} / 2 l\right)(z / h-1)^{2}$ and will not be taken into account here.) Despite the fact that the average internal field acting on the FM film from the direction of the AFM surface is zero, the average magnetization of the FM subsystem is different from zero. It is double-degenerate in direction and is (on average per unit length of the FM film) equals

$$
M \approx M_{0} h^{2} \exp (-L / 2 l)
$$

In reality, the FM film should be broken into superdomains with different directions of the average magnetization in each of them. At the docking of superdomains correct alternation of vortices and antivortices is disturbed and vortex/ vortex or antivortex/antivortex pairs are formed.

\section{MAGNETIC STRUCTURE OF A FM HAVING A STEPWISE DIPOLE WITH $S_{1,2}= \pm 1$ AT THE INTERFACE}

When periodicity in distribution of atomic steps at the interface is disturbed pairs of steps of different signs that are most closely spaced from each other form stepwise dipoles that weakly interact with other dipoles (Fig. 4(b)), and a problem of the structure associated with the dipole of the two DBs emerges. In the case of a stepwise dipole at the FM/AFM interface a pair of gravitating DBs with opposite topological charges emerges in the FM. For this configuration it is easy to find an exact solution of Eq. (7). Note that it is not an exact solution of the original problem, since it does not take into account the boundary conditions (2) and vortex magnetization distribution near the atomic step and does not contain the constant for the exchange interaction through the interface. However, the distribution of the magnetic moments in the thickness on the order of magnetic length correctly describes the true distribution qualitatively.) If we move to a new field variable $\varphi=u / 2$ and new "coordinates" $x=l \xi, z=i l \tau$ we get the well known sinusoidal KleinGordon equation (SGE) $u_{\tau \tau}-u_{\xi \xi}+\sin u=0 .{ }^{14}$ Solution of Eq. (7) for interacting DBs corresponds to a fixed bion solution of $\mathrm{SGE},{ }^{15}$ which translates into the following expression:

$$
\varphi=2 \operatorname{arctg}\left(\frac{\sqrt{1-\nu^{2}}}{\nu} \frac{\operatorname{ch}(\nu(z+h) / l}{\operatorname{ch}\left(\sqrt{1-\nu^{2}} x / l\right)}\right)
$$

where the parameter of solution $\nu$ is determined by the boundary condition at $z=0$ and satisfies the equation

$$
\nu \operatorname{ch}\left(\sqrt{1-\nu^{2}} L / 2 l\right)=\sqrt{1-\nu^{2}} \operatorname{ch}(\nu h / l)
$$

where $L$ is the distance between the fixed DBs at the interface at $z=0$. (Solution of Eq. (24) automatically satisfies the condition at $z=-h$.) The position of domain boundaries is defined by the condition $\varphi=\pi / 2$, and according to the solution of Eq. (24) is determined by the equation

$$
\nu \operatorname{ch}\left(\sqrt{1-\nu^{2}} \frac{L-2 X(z)}{2 l}\right)=\sqrt{1-\nu^{2}} \operatorname{ch}\left(\nu \frac{z+h}{l}\right) .
$$

Consider the case where the size of the step dipole at the interface is significantly less than the thickness of the FM film: $L \ll h$. (However, the film has to remain thin enough $(\ln (h / l)<L / l)$ to avoid formation of closed domains of the type shown in Fig. 4(a)). As will be shown, in this case displacements of the DBs $X(z=-h)=X_{0}$ are on the order of magnitude of the step size of the dipole $X_{0} \sim L$. In this case it follows from the boundary conditions that $\nu \approx X_{0} / h \ll 1$ and from the relationship (25) it follows that

$$
X_{0}=L / 2-l \ln (h / l),
$$

and the equation for the shape of the DB becomes

$$
X(z) \approx\left(\frac{L}{2}-l \ln \frac{h}{l}\right)-X_{0} \frac{z+h}{h} .
$$

This formula is valid in the entire volume of the FM film except in the narrow range $\delta z \sim h\left(l / X_{0}\right) \ll h$ near the free surface of the film, in which formula (28) is modified as follows:

$$
X(z) \approx X_{0}\left[1-\frac{1}{2}\left(\frac{X_{0}}{l}\right)\left(\frac{z+h}{h}\right)^{2}\right]
$$

which corresponds to the formulated boundary conditions. To calculate the total change in magnetization associated with the presence of a surface dipole step, expression (28) can be used, which gives a value of

$$
\delta M=M_{0} h L\left[1+2 \frac{l}{L} \ln \frac{h}{L}\right] .
$$

\section{CONCLUSION}

Under investigation was the distribution of magnetization field in the ferromagnetic layer in contact with a rigid antiferromagnet with a rough interface surface that is modeled by a sequence of atomic steps of alternating signs. The cases of an isolated step associated with a magnetic vortex 
(in the absence of anisotropy in the plane of easy magnetization of a FM) or DB (taking into account this anisotropy), step surface dipole (isolated pair of steps of different signs in the FM/AFM interface plane), and periodic system of alternating surface steps with alternating steps are considered. The change in FM magnetization associated with the roughness of the DB is calculated.

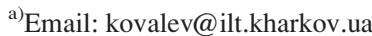

${ }^{1}$ W. H. Maklejohn and C. P. Bean, Phys. Rev. 102, 1413 (1956).

${ }^{2}$ K. B. Vlasov and A. I. Mitsek, Fiz. Met. Metalloved. 14, 487 (1962).

${ }^{3}$ R. L. Stamps and H. E. Camley, Phys. Rev. B 54, 15200 (1996).

${ }^{4}$ R. L. Stamps, H. E. Camley, and R. J. Hocken, Phys. Rev. B 54, 4159 (1996).

${ }^{5}$ M. Kiwi, J. Magn. Magn. Mater. 234, 584 (2001).
${ }^{6}$ J. Nogues and I. K. Schuller, J. Magn. Magn. Mater. 192, 203 (1999).

${ }^{7}$ S. L. Gnatchenko, D. N. Merenkov, A. N. Bludov, V. V. Pishko, Yu. A. Shakhayeva, M. Baran, R. Szymczak, and V. A. Novosad, J. Magn. Magn. Mater. 307, 263 (2006).

${ }^{8}$ A. G. Grechnev, A. S. Kovalev, and M. L. Pankratova, Fiz. Nizk. Temp. 35, 670 (2009) [Low Temp. Phys. 35, 526 (2009)].

${ }^{9}$ A. G. Grechnev, A. S. Kovalev, and M. L. Pankratova, Fiz. Nizk. Temp. 35, 603 (2009) [Low Temp. Phys. 35, 476 (2009)].

${ }^{10}$ O. K. Dudko and A. S. Kovalev, Fiz. Nizk. Temp. 25, 25 (1999) [Low Temp. Phys. 25, 18 (1999)].

${ }^{11}$ O. K. Dudko and A. S. Kovalev, Acta Phys. Pol. A 97, 479 (2000).

${ }^{12}$ O. K. Dudko and A. S. Kovalev, J. Magn. Magn. Mater. 240, 321 (2002).

${ }^{13}$ A. S. Kovalev, A. D. Kondratyuk, and A. I. Landau, Preprint ILTP BASU, No 26-89, Kharkov (1989).

${ }^{14}$ M. J. Ablowitz, D. J. Kaup, A. C. Newell, and H. Segur, Phys. Rev. Lett. 30, 1262 (1973).

${ }^{15}$ A. M. Kosevich and A. S. Kovalev, Introduction to Nonlinear Physical Mechanics (Naukova Dumka, Kiev, 1989).

Translated by D. K. Maraoulaite 